\title{
Relationship Status between Vancomycin Loading Dose and Treatment Failure in Patients with MRSA Bacteremia: It's Complicated
}

\author{
Jessica K. Ortwine - Evan J. Zasowski · Jason M. Pogue • \\ Claudia Hanni - Chris Giuliano - Anthony M. Casapao • \\ Ryan Mynatt · Michael J. Rybak
}

Received: July 23, 2019 / Published online: October 21, 2019

(C) The Author(s) 2019

\section{ABSTRACT}

Introduction: A one-time vancomycin loading dose of $25-30 \mathrm{mg} / \mathrm{kg}$ is recommended in the current iteration of the vancomycin consensus guidelines in order to more rapidly achieve target serum concentrations and hasten clinical improvement. However, there are few clinical data to support this practice, and the extents of its benefits are largely unknown.

Methods: A multicenter, retrospective, cohort study was performed to assess the impact of a

Enhanced Digital Features To view enhanced digital features for this article go to https://doi.org/10.6084/ m9.figshare.9891929.

Electronic supplementary material The online version of this article (https://doi.org/10.1007/s40121019-00268-3) contains supplementary material, which is available to authorized users.

J. K. Ortwine

Department of Pharmacy Services, Parkland Health and Hospital System, Dallas, TX, USA

J. K. Ortwine

University of Texas Southwestern Medical School, Dallas, TX, USA

E. J. Zasowski · C. Hanni · A. M. Casapao ·

M. J. Rybak ( $\square)$

Anti-Infective Research Laboratory, Department of Pharmacy Practice, Eugene Applebaum College of Pharmacy and Health Sciences, Wayne State University, Detroit, MI, USA

e-mail:m.rybak@wayne.edu vancomycin loading dose $(\geq 20 \mathrm{mg} / \mathrm{kg})$ on clinical outcomes and rates of nephrotoxicity in patients with methicillin-resistant Staphylococcus aureus (MRSA) bacteremia. The study matched patients in a 1:1 fashion based on age, Pitt bacteremia score, and bacteremia source. The primary outcome was composite treatment failure (30-day mortality, bacteremia duration $\geq 7$ days after vancomycin initiation, persistent signs and symptoms of infection $\geq 7$ days after vancomycin initiation, or switch to an alternative antimicrobial agent). Secondary outcomes included duration of bacteremia, length of stay post-bacteremia onset, and nephrotoxicity.

Results: A total of 316 patients with MRSA bacteremia were included. Median first doses in the loading dose and non-loading dose groups were $23.0 \mathrm{mg} / \mathrm{kg}$ and $14.3 \mathrm{mg} / \mathrm{kg}$, respectively $(P<0.001)$. No difference was found in

\section{E. J. Zasowski}

Department of Clinical Sciences, College of

Pharmacy, Touro University California, Vallejo, CA, USA

\section{J. M. Pogue}

Department of Pharmacy Services, Sinai-Grace Hospital, Detroit, MI, USA

\section{J. M. Pogue · M. J. Rybak}

Division of Infectious Diseases, Department of Internal Medicine, Wayne State University School of Medicine, Detroit, MI, USA 
composite failure rates between the non-loading dose and loading dose groups $(40.5 \%$ vs. $36.7 \% ; \quad P=0.488)$ or in the incidence of nephrotoxicity $(12.7 \%$ vs. $16.5 \% ; P=0.347)$. While multivariable regression modeling showed receipt of a vancomycin loading dose on a $\mathrm{mg} / \mathrm{kg}$ basis was not significantly associated with composite failure [aOR 0.612, 95\% CI (0.368-1.019)]; post hoc analyses demonstrated that initial doses $\geq 1750 \mathrm{mg}$ were independently protective against failure [aOR 0.506, 95\% CI (0.284-0.902)] without increasing the risk for nephrotoxicity [aOR 0.909, 95\% CI (0.432-1.911)].

Conclusion: These findings suggest that initial vancomycin doses above a certain threshold may decrease clinical failures without increasing toxicity and that weight-based dosing might not be the optimal strategy.

Keywords: Bacteremia; Failure; Loading dose; Nephrotoxicity; Vancomycin

\section{INTRODUCTION}

Various guidelines have suggested different vancomycin dosing and monitoring strategies and it was not until 2009 that the first consensus guideline for the therapeutic monitoring of vancomycin was published [1-4]. The vancomycin guidelines recommend targeting trough concentrations of $15-20 \mathrm{mg} / \mathrm{L}$ for patients with Staphylococcus aureus bacteremia, endocarditis, osteomyelitis, meningitis, or hos-

C. Giuliano

Department of Pharmacy Practice, Eugene

Applebaum College of Pharmacy and Health

Sciences, Wayne State University, Detroit, MI, USA

C. Giuliano

Department of Pharmacy, St. John Hospital and

Medical Center, Detroit, MI, USA

A. M. Casapao

University of Florida College of Pharmacy,

Jacksonville, FL, USA

R. Mynatt · M. J. Rybak

Department of Pharmacy Services, Detroit Receiving

Hospital, Detroit, MI, USA pital-acquired pneumonia and dosing regimens are designed to achieve these target serum exposures at steady-state.

Depending on a patient's renal function, it may take anywhere from 24 to $72 \mathrm{~h}$, or longer, to reach steady-state. To facilitate rapid attainment of goal concentrations, the guidelines recommend a one-time loading dose of $25-30 \mathrm{mg} / \mathrm{kg}$ based off total body weight (TBW) for seriously ill patients [1]. By increasing the likelihood of pharmacokinetic/pharmacodynamic (PK/PD) target attainment early in therapy, this would theoretically improve outcomes in those patients at highest risk of mortality. Although published data demonstrate that achievement of PD targets during the first $48 \mathrm{~h}$ of infection improves outcomes, clinical data showing a direct benefit of vancomycin loading doses are lacking $[5,6]$. Conversely, given previous findings of higher total daily doses being correlated with higher incidence of nephrotoxicity, hypothetical concerns of increased vancomycin-associated nephrotoxicity persist with the weight-based loading dose approach, especially in obese patients [7]. However, this is likely due to resultant supratherapeutic vancomycin exposures in these patients, and there are currently few data to demonstrate an association between vancomycin loading doses and nephrotoxicity $[8,9]$.

Due to the relative paucity of evidence demonstrating advantages to vancomycin loading doses, combined with the concern for increasing the risk for toxic events, continued evaluation of this practice is necessary. The primary objective of this study was to evaluate the effect of administering a one-time, weightbased vancomycin loading dose on clinical outcomes in patients with MRSA bacteremia.

\section{METHODS}

\section{Study Design and Population}

This study was approved with a waiver of informed consent in an expedited review by Wayne State University (IRB \#104312M1E) and by Ascension St. John Hospital (IRB \#785977-6). This study was also performed in accordance 
with the 1964 Helsinki Declaration and its later amendments or comparable ethical standards. This was a retrospective, matched cohort study conducted at two academic health-systems in Southeastern Michigan comprised of 5 acute care hospitals. Patients at least 18 years of age who received vancomycin for treatment of a documented MRSA bacteremia between 2007 and 2013 were eligible for inclusion. Patients were excluded if they received vancomycin for less than $72 \mathrm{~h}$, were pregnant, or had end-stage renal disease or unstable renal function that precluded them from receiving a scheduled vancomycin maintenance dose.

\section{Data Collection}

Data collected included demographics, comorbid conditions, antimicrobial treatment regimens, source of MRSA bacteremia, serum creatinine, Pitt bacteremia score at the time of vancomycin initiation, duration of bacteremia, length of stay, length of vancomycin therapy, vancomycin dosing and trough concentrations, microbiological and clinical cure data, concomitant nephrotoxins, and in-hospital mortality.

\section{Patient Matching}

Patients who received a vancomycin loading dose (first dose $\geq 20 \mathrm{mg} / \mathrm{kg}$ TBW) were matched to those who did not (first dose $<20 \mathrm{mg} / \mathrm{kg}$ TBW) in a $1: 1$ ratio. Patients were matched on the following criteria: age category (18-34 years, $35-64$ years, $\geq 65$ years), Pitt bacteremia score $(<4$ or $\geq 4)$, and bacteremia source risk, as previously defined by Soriano, et al. (low-risk: intravenous catheter, urinary tract, ear-noselarynx, gynecologic; intermediate-risk: osteoarticular, soft-tissue, unknown; and high-risk: endovascular, lower respiratory tract, abdominal, and central nervous system) [10].

\section{Outcome Data and Definitions}

The primary outcome of this study was composite treatment failure defined as the presence of at least one of the following: 30-day mortality (from index culture), bacteremia duration $\geq 7$ days after vancomycin initiation, persistent signs and symptoms of infection [temperature $>38^{\circ} \mathrm{C}$, white blood cells $>12,000 / \mu \mathrm{L}] \geq 7$ days after vancomycin initiation, or switch to an alternative anti-MRSA antimicrobial agent due to treatment failure as determined by treating physician documentation. Patients not meeting criteria for composite failure were considered to be a treatment success. Secondary outcomes included duration of bacteremia, length of stay post-bacteremia onset, and nephrotoxicity. Nephrotoxicity was defined as an increase in serum creatinine (SCr) of greater than $0.5 \mathrm{mg} / \mathrm{dL}$ or at least a $50 \%$ increase from baseline on two consecutive measurements as per the vancomycin dosing and monitoring guidelines, and was assessed starting from the first dose of vancomycin to $72 \mathrm{~h}$ after the final dose [1]. Baseline SCr was the creatinine value immediately preceding the first dose of vancomycin. Vancomycin trough concentration assessment included only initial trough concentrations drawn at steady-state of the maintenance regimen (prior to the 4th or 5th dose). Concomitant nephrotoxins assessed included aminoglycosides, colistin, acyclovir, intravenous (IV) contrast dye, amphotericin, tacrolimus, loop diuretics, non-steroidal antiinflammatory drugs, angiotensin-converting enzyme inhibitors and angiotensin II receptor blockers.

\section{Statistical Analysis}

A sample size of 272 patients, 136 matched pairs, was required to detect a $15 \%$ difference in the primary endpoint using an alpha of 0.05 and power of $80 \%$. For all analyses, a $P$ value $\leq 0.05$ was considered statistically significant. All statistical analyses were performed using SPSS v.24.0 (Armonk, NY, USA).

In the primary analysis, a series of bivariate analyses were performed to compare outcomes between exposure groups, determine factors associated with the primary outcome of composite failure, and determine factors associated with nephrotoxicity. Categorical variables were compared using the $\chi^{2}$ or Fisher's exact test 
while continuous variables were compared using the Student's $t$ test or Mann-Whitney $U$ test. Multivariable regression analyses were then performed to examine the independent association between loading dose and composite failure as well as loading dose and nephrotoxicity. Loading dose, along with all variables associated with the outcome of interest at a $P$ value $<0.2$ with biologic plausibility were entered into conditional logistic regression models simultaneously and removed in a backward, stepwise fashion, being retained in the logistic regression model if the $P$ value for the likelihood ratio test for their removal was $<0.1$. Because loading dose was the exposure of interest, it was forced to remain in final step of regression models even if no statistical association was observed. Model fit was assessed with the Hosmer-Lemmeshow goodness-of-fit test; models with a non-significant result were considered adequate. Multicollinearity of candidate regression models was assessed via the variance inflation factor, with values between 1 and 5 considered acceptable.

\section{Post-Hoc Analyses}

Based on the unequal distribution of TBW between the loading dose and non-loading dose groups, the lack of association between first dose measured in $\mathrm{mg} / \mathrm{kg}$, and the mild association between first dose in $\mathrm{mg}$ and outcome $(P=0.12)$ in the primary analysis, post hoc exploratory analyses were performed to further examine the association between initial vancomycin dose, measured in $\mathrm{mg}$, and outcome. Classification and regression tree (CART) analysis was performed to derive a threshold in the distribution of initial vancomycin dose, modeled continuously, where the incidence of composite failure was most disproportionate. After identifying this threshold, it was entered into regression analysis in place of loading dose to examine its independent association with composite failure.

Furthermore, given both the obesity imbalance between treatment groups and the unexpected finding of obesity being protective against treatment failure, further analyses were performed to ensure the lack of association between a weight-based loading dose strategy and outcome was not an artifact of obese patients being less likely to receive first doses $\geq 20 \mathrm{mg} / \mathrm{kg}$. This was accomplished by two separate methods. First, failure rates were compared in patients receiving loading doses to those who did not as a function of body mass index (BMI) classification (i.e., underweight, normal/overweight, and obese). This same analysis was also performed in the different BMI classifications for the CART-defined milligrambased (non-weight-based) first dose cutoff for success. Secondly, the multivariate models for independent predictors of failure were performed excluding the obese patients population $(n=62)$ to assess the impact on the association between loading dose or the CART-defined cutoff on treatment failure in the rest of the cohort.

\section{RESULTS}

\section{Patient Population}

A total of 316 patients constituting 158 matched pairs were included in the final analysis. The baseline demographics of the patients were similar in each group, although patients who did not receive loading doses had significantly higher TBW and prevalence of obesity (Table 1). The most common source of MRSA bacteremia was skin and soft tissue infection. Over onethird of the patients in each group required admission to an intensive care unit (ICU) at some point during admission, but overall Pitt bacteremia scores remained low in both groups. Vancomycin minimum inhibitory concentration (MIC) was available for 292 of the isolates, with an $\mathrm{MIC}_{50}$ of $1 \mathrm{mg} / \mathrm{L}$ (range $0.5-2 \mathrm{mg} / \mathrm{L}$ ). Among patients in the loading dose group, the median (IQR) initial dose was $23.0 \mathrm{mg} / \mathrm{kg}$ (21.4-25.0) equating to $1500 \mathrm{mg}$ (IQR 1500-2000). This was significantly greater than the initial dose of $14.3 \mathrm{mg} / \mathrm{kg}$ (IQR 12.2-17.1) or $1000 \mathrm{mg}$ (IQR 1000-1250) received by patients in the non-loading dose group $(P<0.001$ for both comparisons). Although loading dose patients received a higher median (IQR) 
Table 1 Bivariate comparisons between non-loading dose and loading dose patients

\begin{tabular}{lll}
\hline Characteristic & No loading & $\begin{array}{l}\text { Loading dose } P \text { value } \\
(n=158) \\
\text { dose } \\
(n=158)\end{array}$ \\
\hline
\end{tabular}

Demographics

Age, mean (SD)

$\begin{array}{lll}57.4(15.3) & 56.8(17.4) & 0.721 \\ 74(46.8) & 72(45.6) & 0.821 \\ & & 1.000 \\ 118(74.7) & 118(74.7) & \\ 40(25.3) & 40(25.3) & \end{array}$

Ascension St. John Hospital

$40(25.3) \quad 40(25.3)$

Clinical characteristics

Prior hospitalization (30 days)

$47(29.7)$

49 (31.0)

0.807

Prior $S$. aureus infection (30 days)

4 (2.5)

$6(3.8)$

0.750

Total body weight $(\mathrm{kg})$

$75(64-90.8)$

$70(61-79.8)$

0.001

Obesity $\left(\geq 30 \mathrm{~kg} / \mathrm{m}^{2}\right)$

42 (26.6)

$20(12.7)$

0.002

Intravenous drug use

40 (25.3)

$43(27.2)$

0.701

Diabetes

$50(31.6)$

35 (22.2)

0.057

Cerebrovascular accident

$30(19.0)$

17 (10.8)

0.040

Cirrhosis

6 (3.8)

$1(0.6)$

0.121

Malignancy

$12(7.6)$

$16(10.1)$

0.428

HIV/AIDS

$6(3.8)$

$12(7.6)$

0.145

Creatinine clearance $(\mathrm{mL} / \mathrm{min})$

72.5

75.9

0.981

$(52.7-102.5)$

(48.6-107.6)

Concomitant nephrotoxins

$\begin{array}{lll}118(74.7) & 112(70.9) & 0.448 \\ 1(0-2) & 1(0-2) & 0.203 \\ 0(0-1) & 0(0-1) & 0.259 \\ 1(0-3) & 1(0-2) & 0.166 \\ 34(21.5) & 54(34.2) & 0.012 \\ & & 0.465\end{array}$

Primary bacteremia source

$5(3.2)$

$17(10.8)$

Bone/joint

$20(12.7)$

$16(10.1)$

Intravenous catheter

$15(9.5)$

$15(9.5)$

Urinary

3 (1.9)

2 (1.3)

Lower respiratory tract

$27(17.1)$

24 (15.2)

Skin/soft tissue

$46(29.1)$

44 (27.8) 
Table 1 continued

\begin{tabular}{|c|c|c|c|}
\hline Characteristic & $\begin{array}{l}\text { No loading } \\
\text { dose } \\
(n=158)\end{array}$ & $\begin{array}{l}\text { Loading dose } \\
(n=158)\end{array}$ & $P$ value \\
\hline Infective endocarditis & $16(10.1)$ & $17(10.8)$ & \\
\hline Unknown & $22(13.9)$ & $20(12.7)$ & \\
\hline Other & $4(2.5)$ & $3(1.9)$ & \\
\hline \multicolumn{4}{|l|}{ Treatment information } \\
\hline Time to vancomycin (days) & $1(0-1)$ & $0(0-1)$ & 0.396 \\
\hline First dose (mg) & $\begin{array}{l}1000 \\
(1000-1250)\end{array}$ & $\begin{array}{l}1500 \\
\quad(1500-2000)\end{array}$ & $<0.001$ \\
\hline First dose $(\mathrm{mg} / \mathrm{kg})$ & $14.3(12.2-17.1)$ & $23.0(21.4-25.0)$ & $<0.001$ \\
\hline Initial maintenance dose $(\mathrm{mg})$ & $\begin{array}{l}1000 \\
(1000-1250)\end{array}$ & $\begin{array}{l}1000 \\
\quad(1000-1250)\end{array}$ & 0.470 \\
\hline Initial maintenance dose $(\mathrm{mg} / \mathrm{kg})$ & $13.8(11.7-16.6)$ & $15.7(13.2-19.2)$ & $<0.001$ \\
\hline $\begin{array}{l}\text { Initial trough concentration during first } 72 \mathrm{~h}(\mathrm{mg} / \mathrm{L})(n=101 \text { non-LD; } \\
105 \mathrm{LD})\end{array}$ & $12.8(9.4-16.3)$ & $14.4(10.6-17.9)$ & 0.081 \\
\hline Initial trough concentration $\geq 15 \mathrm{mg} / \mathrm{L}(n=101$ non-LD; $105 \mathrm{LD})$ & $35(34.7)$ & $48(45.7)$ & 0.106 \\
\hline Initial trough concentration $\geq 10 \mathrm{mg} / \mathrm{L}(n=101$ non-LD; $105 \mathrm{LD})$ & $72(71.3)$ & $81(77.1)$ & 0.336 \\
\hline Inpatient duration of therapy (days) & $8(5-12)$ & $8(5-12)$ & 0.324 \\
\hline \multicolumn{4}{|l|}{ Outcomes } \\
\hline Bacteremia duration (days) & $4(2-6)$ & $3(2-5)$ & 0.287 \\
\hline ICU LOS & $6(3-16)$ & $6(2-10)$ & 0.181 \\
\hline LOS post-bacteremia & $12(8-18)$ & $10(7-16)$ & 0.185 \\
\hline Composite failure & $64(40.5)$ & $58(36.7)$ & 0.488 \\
\hline 30-day mortality & $14(8.9)$ & $18(11.4)$ & 0.456 \\
\hline Bacteremia duration $\geq 7$ days & $21(13.3)$ & $27(17.1)$ & 0.347 \\
\hline Persistent signs/symptoms $\geq 7$ days & $30(19.0)$ & $29(18.4)$ & 0.885 \\
\hline Switch to alternate agent due to treatment failure & $31(19.6)$ & $23(14.6)$ & 0.232 \\
\hline Nephrotoxicity & $20(12.7)$ & $26(16.5)$ & 0.339 \\
\hline
\end{tabular}

Data presented at $n(\%)$ or median (IQR) unless otherwise specified

$H I V / A I D S$ human immunodeficiency virus/acquired immune deficiency syndrome, $L O S$ length of stay, $I C U$ intensive care unit, $L D$ loading dose

maintenance dose measured in $\mathrm{mg} / \mathrm{kg}[15.7$ (13.2-19.2) vs. $13.8 \quad(11.7-16.6) \quad \mathrm{mg} / \mathrm{kg}$, $P<0.001]$, maintenance doses measured in $\mathrm{mg}$ were comparable between groups [1000
(1000-1250) vs. 1000 (1000-1250) mg, $P=0.470]$. For patients who had serum trough concentrations drawn within the first $72 \mathrm{~h}$ of therapy ( $n=105$ loading dose, 101 non-loading 
dose), no significant differences were observed in median first trough concentrations or the proportion of patients with initial trough concentrations $\geq 15 \mathrm{mg} / \mathrm{L}$.

\section{Outcomes}

In bivariate analysis, there was no difference in composite failure between patients in the nonloading dose and loading dose groups $(40.5 \%$ vs. $36.7 \% ; P=0.488)$. The incidence of specific components of composite failure in each group can be seen in Table 1; however, no difference was observed between any of the individual components. Nephrotoxicity occurred in 21 patients in the non-loading dosing group and 27 patients in the loading dose group $(12.7 \%$ vs. $16.5 \% ; P=0.339$ ). The results of the final multivariable regression model for composite failure are displayed in Table 2. There was no association between receipt of a vancomycin loading dose and the primary outcome of composite failure [aOR 0.612 (95\% CI $(0.368-1.019)$ ]. Infective endocarditis [aOR 3.583 (1.599-8.029)] and ICU level of care at vancomycin initiation [aOR 4.145 (2.389-7.191)] were independently associated with composite failure while intravenous catheter source [aOR $0.327(0.115-0.929)]$ and obesity [aOR 0.497 (0.255-0.965)] were protective against treatment failure. The results of the final multivariable regression model for nephrotoxicity are displayed in Table 3. Receipt of a vancomycin loading dose was not associated with risk of nephrotoxicity [aOR 1.295 (0.657-2.553)].

\section{Post-Hoc Analyses}

As described above, post-hoc CART analysis on initial vancomycin dose (mg) was performed to determine if a milligram-based cutoff predicting success could be identified. This analysis unveiled a threshold of $\geq 1750 \mathrm{mg}$, above which the proportion of patients experiencing composite failure (Supplemental Table 1) was significantly lower $[25 / 86 \quad(29.1 \%)$ receiving $\geq 1750 \mathrm{mg}$ vs. 97/230 (42.2\%) receiving $<1750 \mathrm{mg}, P=0.033$ ]. CART analysis was unable to determine a $\mathrm{mg} / \mathrm{kg}$-based cutoff. In multivariable regression analyses including initial dose $\geq 1750 \mathrm{mg}$ in place of vancomycin loading dose, doses $\geq 1750 \mathrm{mg}$ were independently protective against failure [aOR 0.506 (0.284-0.902)] and obesity was no longer independently protective against failure (Table 2 ).

When treatment failure rates were assessed for both exposure cutoffs (presence/absence of loading dose of $\geq 20 \mathrm{mg} / \mathrm{kg}$ and presence/absence of first dose of $\geq 1750 \mathrm{mg}$ ) as a function of BMI category, failure rates were lowest for obese patients and there was no association between first dose and outcome in obese patients (Table 4). Initial dose $\geq 1750 \mathrm{mg}$ was associated with decreased failure in the normal/ overweight cohort (31.0\% vs. $47.5 \% ; P=0.032)$. No such association was seen with loading doses $\geq 20 \mathrm{mg} / \mathrm{kg}$ in normal/overweight patients, with failure seen in $42.4 \%$ and $44.1 \%$, respectively $(P=0.89)$. Furthermore, when all obese patients were removed from the cohort, the magnitude of the adjusted odds ratios in the multivariate models for loading dose [aOR $0.697(0.406-1.196)]$ and first dose $\geq 1750 \mathrm{mg}$ [aOR $0.561(0.287-1.094)$ ] and treatment failure were similar to that of the overall cohort, and only failed to reach significance for first dose $\geq 1750 \mathrm{mg}$ due to wider confidence intervals due to the decrease in sample size. Importantly, when initial dose of $\geq 1750 \mathrm{mg}$ was placed in the model for nephrotoxicity instead of vancomycin loading dose, no association between this dose and toxicity was demonstrated [aOR 0.909 (0.432-1.911)].

\section{DISCUSSION}

In the present study, there was no significant correlation between vancomycin loading dose and clinical success when the loading dose was assessed in the traditional $(\mathrm{mg} / \mathrm{kg})$ sense. It is noteworthy, however, that, when controlling for other factors, there was a signal between a $\mathrm{mg} / \mathrm{kg}$-based first dose and improved outcome that failed to reach statistical significance. Further investigation revealed that first dose, when looked at on a milligram basis alone, did have a significant impact on clinical outcomes. 
Table 2 Logistic regression for factors associated with composite failure

Variable Unadjusted OR (95\% CI)

Adjusted OR (95\% CI)

Primary analysis: loading dose $\geq 20 \mathrm{mg} / \mathrm{kg}^{\mathrm{a}}$

ICU at vancomycin initiation

$$
\begin{aligned}
& 3.489(2.091-5.822) \\
& 3.660(1.705-7.855) \\
& 0.289(0.107-0.776) \\
& 0.486(0.261-0.904) \\
& 0.852(0.541-1.341) \\
& 4.103(0.783-21.487) \\
& 2.013(1.100-3.685) \\
& 1.211(1.056-1.389) \\
& 1.015(1.000-1.029) \\
& 0.301(0.085-1.062) \\
& 0.382(0.106-1.384) \\
& 0.849(0.642-1.122) \\
& 0.968(0.926-1.012)
\end{aligned}
$$$$
4.145(2.389-7.191)
$$

Infective endocarditis source

$3.583(1.599-8.029)$

Intravenous catheter source

0.327 (0.115-0.929)

Obesity

$0.497(0.255-0.965)$

Loading dose

$0.612(0.368-1.019)$

Cirrhosis

Lower respiratory tract source

Pitt bacteremia score

Age

HIV/AIDS

Unknown source

Time to vancomycin

LOS pre-bacteremia

Post-hoc analysis: initial dose $\geq 1750 \mathrm{mg}^{\mathrm{b}}$

ICU at vancomycin initiation

$$
\begin{aligned}
& 3.489(2.091-5.822) \\
& 3.660(1.705-7.855) \\
& 0.289(0.107-0.776) \\
& 0.562(0.329-0.958) \\
& 0.382(0.106-1.384) \\
& 4.103(0.783-21.487) \\
& 2.013(1.100-3.685) \\
& 1.211(1.056-1.389) \\
& 1.015(1.000-1.029) \\
& 0.486(0.261-0.904) \\
& 0.301(0.085-1.062) \\
& 0.849(0.642-1.122) \\
& 0.968(0.926-1.012)
\end{aligned}
$$$$
\text { HIV/AIDS }
$$

Time to vancomycin

$$
\begin{aligned}
& 4.127(2.385-7.140) \\
& 3.353(1.500-7.495) \\
& 0.293(0.104-0.822) \\
& 0.506(0.284-0.902) \\
& 0.342(0.090-1.303) \\
& - \\
& - \\
& - \\
& -
\end{aligned}
$$$$
\text { LOS pre-bacteremia }
$$

$I C U$ intensive care unit, $H I V / A I D S$ human immunodeficiency virus/acquired immune deficiency syndrome, LOS length of stay

${ }^{a}$ Hosmer-Lemeshow goodness of fit test $P=0.628$; variance inflation factor $1-5$ for all variables included at model entry

b Hosmer-Lemeshow goodness of fit test $P=0.762$; variance inflation factor $1-5$ for all variables included at model entry 
Table 3 Logistic regression for factors associated with nephrotoxicity

\begin{tabular}{lll}
\hline Variable & Unadjusted OR (95\% CI) & Adjusted OR (95\% CI) \\
\hline Primary analysis: loading dose $\geq 20 \mathrm{mg} / \mathrm{kg}^{\mathrm{a}}$ & & \\
ICU at vancomycin initiation & $3.154(1.600-5.992)$ & $2.658(1.332-5.305)$ \\
Concomitant IV contrast dye & $2.165(1.147-4.087)$ & $2.329(1.202-4.511)$ \\
Concomitant loop diuretic & $2.630(1.365-5.066)$ & $2.189(1.075-4.458)$ \\
Loading dose & $1.359(0.724-2.552)$ & $1.295(0.657-2.553)$ \\
Concomitant aminoglycoside & $2.518(0.904-5.151)$ & - \\
Malignancy & $2.218(0.848-5.338)$ & - \\
Male sex & $0.641(0.336-1.221)$ & - \\
Post-hoc analysis: initial dose $\geq 1750 \mathrm{mg}{ }^{\mathrm{b}}$ & & $2.826(1.435-5.567)$ \\
ICU at vancomycin initiation & $3.154(1.600-5.992)$ & $2.337(1.206-4.529)$ \\
Concomitant IV contrast dye & $2.165(1.147-4.087)$ & $2.072(1.035-4.148)$ \\
Concomitant loop diuretic & $2.630(1.365-5.066)$ & $0.909(0.432-1.911)$ \\
Initial dose $\geq 1750$ mg & $0.935(0.459-1.902)$ & - \\
Concomitant aminoglycoside & $2.518(0.904-5.151)$ & - \\
Malignancy & $2.218(0.848-5.338)$ & - \\
Male sex & $0.641(0.336-1.221)$ & \\
\hline
\end{tabular}

$I C U$ intensive care unit

${ }^{a}$ Hosmer-Lemeshow goodness of fit test $P=0.310$; variance inflation factor $1-5$ for all variables included at model entry

b Hosmer-Lemeshow goodness of fit test $P=0.977$; variance inflation factor $1-5$ for all variables included at model entry

Specifically, first doses of at least $1750 \mathrm{mg}$ were protective against composite failure.

While first doses $\geq 1750 \mathrm{mg}$ being predictive of success and loading doses $\geq 20 \mathrm{mg} / \mathrm{kg}$ having no association with failure is an interesting and important finding, a potential confounder of this dataset was that obesity was found to be protective against composite failure. One possible explanation for the above finding is that obese patients may have received higher first doses (in $\mathrm{mg}$ ) despite the dose not meeting the arbitrary $\mathrm{mg} / \mathrm{kg}$ definition of a loading dose. In order to ensure this patient group did not drive the lack of association between $\mathrm{mg} / \mathrm{kg}$-based loading dose and outcomes, multiple additional analyses were performed, the results of which demonstrate that obesity itself is unlikely to have obscured any relationship, and that the association between milligram-based (flat) first doses and outcomes is truly stronger than $\mathrm{mg} /$ kg-based loading doses.

First, Table 4 clearly demonstrates that obese patients were less likely to experience composite failure compared to other patients in the cohort regardless of either $\mathrm{mg} / \mathrm{kg}$ or flat first dose cutoff $(20 \mathrm{mg} / \mathrm{kg}$ or $1750 \mathrm{mg})$. One possible explanation for this finding is that obese patients were less likely to have a "high-risk" source of MRSA bacteremia then non-obese patients $(30 \%$ vs. $18 \% ; P=0.08$; data not shown). Secondly, to further ensure that obesity was not confounding an association with weight-based dosing we performed the same regression analyses with obese patients removed from the cohort. Without this group of patients, the adjusted odds ratios for composite failure 
Table 4 Association between first dose and composite failure stratified by body mass index category

\begin{tabular}{|c|c|c|c|}
\hline & First dose $<20 \mathrm{mg} / \mathrm{kg}$ & First dose $\geq 20 \mathrm{mg} / \mathrm{kg}$ & $P$ value \\
\hline \multicolumn{4}{|c|}{ Composite failure: primary analysis } \\
\hline Underweight $^{a}$ & $7 / 14(50)$ & $4 / 20(20)$ & 0.14 \\
\hline Normal/overweight ${ }^{\mathrm{b}}$ & $45 / 102(44.1)$ & $50 / 118(42.4)$ & 0.89 \\
\hline \multirow[t]{2}{*}{ Obese $^{c}$} & $12 / 42(28.6)$ & $4 / 20(20)$ & 0.55 \\
\hline & First dose $<1750 \mathrm{mg}$ & First dose $\geq 1750 \mathrm{mg}$ & $P$ value \\
\hline \multicolumn{4}{|c|}{ Composite failure: post hoc analysis } \\
\hline Underweight $^{a}$ & $11 / 34(32.4)$ & 0 & - \\
\hline Normal/overweight ${ }^{\mathrm{b}}$ & $77 / 162(47.5)$ & $18 / 58(31)$ & 0.03 \\
\hline Obese $^{c}$ & $9 / 34(26.5)$ & $7 / 28(25)$ & 1.00 \\
\hline
\end{tabular}

and first doses in $\mathrm{mg} / \mathrm{kg}$ and $\geq 1750 \mathrm{mg}$ were similar to those for the entire cohort. Importantly, the adjusted odds ratio for $\mathrm{mg} / \mathrm{kg}$-based loading dose and treatment failure actually increased slightly when obese patients were removed from the cohort. If these patients were truly obscuring an association, it would be expected that the adjusted odds ratios would decrease (or at the least stay the same) when these patients were removed from the cohort, even if they failed to reach statistical significance due to sample size.

Additional stratified analyses further support the association between initial doses of $1750 \mathrm{mg}$ or greater rather than weight-based loading doses as the true driver in clinical success in our cohort. The benefit of a first dose $\geq 1750 \mathrm{mg}$ was primarily observed in normal/overweight individuals, which was the predominate weight class of the patients in this study. Conversely, when assessing weight-based doses in this same cohort of patients, no signal of an association was identified with first doses $\geq 20 \mathrm{mg} / \mathrm{kg}$. Interestingly, the only weight category that suggested a potential benefit from a weightbased loading dose was those who were underweight. In this cohort of patients, failure was seen in $4 / 20(20 \%)$ of patients who received a weight-based loading dose compared to $7 / 14$ (50\%) of those who did not. While this association failed to reach significance due to small numbers, it is logical that this would be the cohort where a weight-based dose might show the most benefit as it would allow patients in this group to receive a dose closer to the threshold $\mathrm{mg}$ dose. However, given the small numbers, and the fact that no patient in this weight category received a dose of at least $1750 \mathrm{mg}$, we were unable to fully assess the threshold in this patient population. Finally, while the CART analysis was able to identify $1750 \mathrm{mg}$ as a flat-dose threshold, it was unsuccessful at identifying an $\mathrm{mg} / \mathrm{kg}$ cutoff value associated with composite failure. Taken together, these data support the finding that a flat, milligram-based first dose, rather than a $\mathrm{mg} / \mathrm{kg}$ based one, may improve patient outcomes.

It is important to note that the finding that doses $\geq 1750 \mathrm{mg}$ decreased treatment failure should not to be interpreted as a threshold for what a loading dose should be, but more so as a proof of concept that there is an association between initial vancomycin dose and clinical outcome, and that that dose might not be best determined by a patient's weight. Finding an association between first dose and outcome is 
not surprising given the wealth of evidence demonstrating the importance of attaining adequate vancomycin exposure on day 1 and 2 of therapy on improving outcomes $[8,9,11]$. While this study assessed both first dose and maintenance dose regimens, it did not assess the timing between those doses and thus cannot assess day 1 area under the time-versusconcentration curve (AUC). Therefore, the 1750-mg dose identified by CART analysis in this study cannot be extrapolated to the greater population as the total exposure on day 1 associated with this value could not be ascertained.

MIC values were available for the majority of the MRSA isolates; however, this information was not included in any of the analyses given the known inaccuracies with the various testing methodologies. Vancomycin MIC values for MRSA performed using automated susceptibility testing have been shown to vary from the Clinical Laboratory Standards Institute broth microdilution method by \pm 1 dilution, whereas MIC testing via Etest methodology tended to produce MIC values 1-2 dilutions higher than broth microdilution [12]. As the vast majority of MRSA isolates have a MIC value of $1 \mathrm{mg} / \mathrm{L}$, this variable likely had little impact on the outcomes of this study [13].

While the association between a flat milligram-based first dose and not a mg/kg-based dose and clinical failure was novel and unexpected, it should not be a surprise. The only pharmacokinetic parameter impacted by the first dose is peak serum concentration $\left(C_{\max }\right)$, which is dependent not only on the dose but also on the volume of distribution $\left(V_{\mathrm{d}}\right)$. It is well established that $V_{\mathrm{d}}$ is lower $(0.26-0.56 \mathrm{~L} / \mathrm{kg}$ of total body weight) in obese patients than the $0.7 \mathrm{~L} / \mathrm{kg}$ cited for normal weight individuals [14-17]. Given this information, it makes sense that, while obese patients may need a higher first dose due to a higher overall $V_{\mathrm{d}}$, that dose does not need to increase proportionally with weight because $V_{\mathrm{d}}$ is not increasing proportionally. This finding is further supported by Reynolds et al. [18], who reported that obese patients who received vancomycin dosed at $10 \mathrm{mg} / \mathrm{kg} /$ dose achieved more therapeutic concentrations and fewer supratherapeutic troughs than those who received $15 \mathrm{mg} / \mathrm{kg} / \mathrm{dose}$. Similarly, AUC is dependent on initial dose as well as on drug clearance. Vancomycin clearance is best estimated using the adjusted body weight of an obese patient [19]. Using this information, a $\mathrm{mg} / \mathrm{kg}$ vancomycin dose based on total body weight would likely overshoot the AUC target, given the disproportionate increase in clearance. This provides further support that a flat dose may be a more appropriate dosing strategy in this patient population. As previously stated, we were unable to validate this assumption, given the absence of maintenance dosing timing as well as lack of day 1 AUC data.

The clinical failure rate of around $40 \%$ in each group is consistent with failure rates documented in other studies using trough-based vancomycin dosing for the treatment of MRSA bacteremia [20-22]. Although a few analyses have assessed the impact of PK determined loading doses on day 1 AUC or trough target attainment, only one study has assessed the impact of loading doses on outcomes [23-26]. Wesolek and colleagues [26] performed a retrospective cohort study to evaluate the impact of initial vancomycin doses on resolution of systemic inflammatory response syndrome (SIRS) criteria in patients with sepsis secondary to MRSA bacteremia. Patients who received a first dose of vancomycin $\geq 20 \mathrm{mg} / \mathrm{kg}(n=37)$ experienced resolution of SIRS criteria within $67 \mathrm{~h}$, on average, compared to $109 \mathrm{~h}$ in patients receiving first doses of vancomycin $<20 \mathrm{mg} / \mathrm{kg}$ $(n=87)$ and Cox proportional hazard modeling showed a faster resolution of SIRS in the first dose $\geq 20 \mathrm{mg} / \mathrm{kg}$ group [HR = $1.72(1.09-2.73)$ ]. It was hypothesized that this was likely due to more rapid achievement of therapeutic serum vancomycin concentrations among patients receiving the higher first dose; however, day 1 exposures were not reported and other dosing strategies were not assessed.

A common cause for hesitation with higher first doses is a perceived risk for increased rates of acute kidney injury. In this regard, the data presented in this analysis are extremely encouraging as neither a first dose $\geq 20 \mathrm{mg} / \mathrm{kg}$ nor a first dose of $\geq 1750 \mathrm{mg}$ was a risk factor for of development of nephrotoxicity. These findings are further supported by a study 
performed by Rosini et al. [27], which compared rates of nephrotoxicity ( 2 serial $\mathrm{SCr}$ values of $\geq 0.5 \mathrm{mg} / \mathrm{dL}$ from baseline or an increase of $\geq 50 \%$ ) and acute kidney injury (AKI; a single SCr increase of $\geq 0.5 \mathrm{mg} / \mathrm{dL}$ or $\geq 50 \%$ increase from baseline) among patients receiving vancomycin with first doses $>20 \mathrm{mg} / \mathrm{kg}$ and $\leq 20 \mathrm{mg} / \mathrm{kg}$. In this analysis, nephrotoxicity and AKI actually occurred less frequently in patients who received a first dose $>20 \mathrm{mg} / \mathrm{kg}$ compared to patients receiving $\leq 20 \mathrm{mg} / \mathrm{kg}$ (5.8\% vs. $11.1 \% ; P<0.001$ for nephrotoxicity; and $7.5 \%$ vs. $12.8 \% ; P<0.001$ for AKI). Taken together, these data support the safety of vancomycin loading doses to optimize patient outcomes.

The findings of this study are not without limitations. First, this study was retrospective in nature, which could lead to information bias. Although incomplete documentation in the medical record can make it difficult to accurately measure outcomes retrospectively, we constructed a primary composite failure outcome based largely on readily available objective criteria, such as mortality and bacteremia duration which should limit the impact this has on the outcomes assessed. Secondly, while the guideline-recommended loading dose is $25-30 \mathrm{mg} / \mathrm{kg}$, all first doses above $20 \mathrm{mg} / \mathrm{kg}$ were considered a loading dose in this study. This was done to capture patients intended to receive a loading dose, but who may have received slightly less than the guideline recommendation due to the common practice of doserounding to the nearest $250 \mathrm{mg}$ increment. Additionally, as one component of the failure definition was a switch from vancomycin to alternative agents, prescribing bias in therapeutic preference could come into play. Encouragingly, there was no difference in this outcome between any of our groups, and this did not drive the differences in composite failure seen in this study. Although the study included two health systems, the vancomycin dosing practices at these institutions may not be reflective of the diverse range of practices employed. In particular, this study included a large proportion of patients who were not critically ill, the area where loading doses are theorized to provide the greatest benefit. As such, the results of this study may not fully capture the impact of administering a loading dose in this population. Finally, as previously discussed, while an association between vancomycin first doses and clinical failure was observed, evaluation of the maintenance dose was not performed and carries with it multiple implications. While maintenance doses and steady-state vancomycin troughs were similar between the cohorts, timing of initiation of these maintenance regimens and the resulting $\mathrm{AUC}_{0-24}$ exposures, were not assessed. An inappropriately timed maintenance regimen (i.e., too great an interval between administration of the first dose and the maintenance regimen) has the potential to derail any theoretical benefit gained by administering a loading dose.

\section{CONCLUSION}

To date, this is one of the only studies to examine the association between vancomycin loading doses, clinical outcomes and nephrotoxicity in patients with MRSA bacteremia. No significant difference in efficacy or toxicity was seen between those patients who received loading doses $\geq 20 \mathrm{mg} / \mathrm{kg}$ TBW and those who received a smaller initial dose. This study found that initial doses $\geq 1750 \mathrm{mg}$ were associated with clinical success; however, due to the aforementioned limitations, this should not be interpreted as the definitive first dose threshold. Rather, these finding highlights that there is an association between first dose and clinical outcome and that, contrary to previous belief, this first dose may not need to be an $\mathrm{mg} / \mathrm{kg}$-based dose. Additional studies combining first dose data, day 1 exposures, and clinical outcomes are needed to fully discern the impact of vancomycin first doses.

\section{ACKNOWLEDGEMENTS}

Funding. No funding or sponsorship was received for this study or publication of this article. 
Authorship. All named authors meet the International Committee of Medical Journal Editors (ICMJE) criteria for authorship for this article, take responsibility for the integrity of the work as a whole, and have given their approval for this version to be published.

Disclosures. Jessica K. Ortwine, Evan J. Zasowski, Jason M. Pogue, Claudia Hanni, Chris Giuliano, Anthony M. Casapao, Ryan Mynatt, and Michael J. Rybak have nothing to disclose.

Compliance with Ethics Guidelines. This study was approved with a waiver of informed consent in an expedited review by Wayne State University (IRB \#104312M1E) and by Ascension St. John Hospital (IRB \#785977-6). This study was also performed in accordance with the 1964 Helsinki Declaration and its later amendments or comparable ethical standards.

Open Access. This article is distributed under the terms of the Creative Commons Attribution-NonCommercial 4.0 International License (http://creativecommons.org/licenses/ by-nc/4.0/), which permits any noncommercial use, distribution, and reproduction in any medium, provided you give appropriate credit to the original author(s) and the source, provide a link to the Creative Commons license, and indicate if changes were made.

\section{REFERENCES}

1. Rybak M, Lomaestro B, Rotschafer JC, Moellering R Jr, Craig W, Billeter $\mathrm{M}$, et al. Therapeutic monitoring of vancomycin in adult patients: a consensus review of the American Society of Health-System Pharmacists, the Infectious Diseases Society of America, and the Society of Infectious Diseases Pharmacists. Am J Health Syst Pharm. 2009;66(1):82-98.

2. Karam CM, McKinnon PS, Neuhauser MM, Rybak MJ. Outcome assessment of minimizing vancomycin monitoring and dosing adjustments. Pharmacotherapy. 1999;19(3):257-66.

3. Kalil AC, Metersky ML, Klompas M, Muscedere J, Sweeney DA, Palmer LB, et al. Management of adults with hospital-acquired and ventilator- associated pneumonia: 2016 clinical practice guidelines by the Infectious Diseases Society of America and the American Thoracic Society. Clin Infect Dis. 2016;63(5):e61-111.

4. Liu C, Bayer A, Cosgrove SE, Daum RS, Fridkin SK, Gorwitz RJ, et al. Clinical practice guidelines by the Infectious Diseases Society of America for the treatment of methicillin-resistant Staphylococcus aureus infections in adults and children. Clin Infect Dis. 2011;52(3):e18-55.

5. Lodise TP, Drusano GL, Zasowski E, Dihmess A, Lazariu V, Cosler L, et al. Vancomycin exposure in patients with methicillin-resistant Staphylococcus aureus bloodstream infections: how much is enough? Clin Infect Dis. 2014;59(5):666-75.

6. Casapao AM, Lodise TP, Davis SL, Claeys KC, Kullar $\mathrm{R}$, Levine DP, et al. Association between vancomycin day 1 exposure profile and outcomes among patients with methicillin-resistant Staphylococcus aureus infective endocarditis. Antimicrob Agents Chemother. 2015;59(6):2978-85.

7. Lodise TP, Lomaestro B, Graves J, Drusano GL. Larger vancomycin doses (at least four grams per day) are associated with an increased incidence of nephrotoxicity. Antimicrob Agents Chemother. 2008;52(4):1330-6.

8. Zasowski EJ, Murray KP, Trinh TD, Finch NA, Pogue JM, Mynatt RP, et al. Identification of vancomycin exposure-toxicity thresholds in hospitalized patients receiving intravenous vancomycin. Antimicrob Agents Chemother. 2017;62(1):e01684e17.

9. Neely MN, Youn G, Jones B, Jelliffe RW, Drusano GL, Rodvold KA, et al. Are vancomycin trough concentrations adequate for optimal dosing? Antimicrob Agents Chemother. 2014;58(1):309-16.

10. Soriano A, Marco F, Martinez JA, Pisos E, Almela M, Dimova VP, et al. Influence of vancomycin minimum inhibitory concentration on the treatment of methicillin-resistant Staphylococcus aureus bacteremia. Clin Infect Dis. 2008;46(2):193-200.

11. Zelenitsky S, Rubinstein E, Ariano R, Iacovides $H$, Dodek P, Mirzanejad Y, et al. Vancomycin pharmacodynamics and survival in patients with methicillin-resistant Staphylococcus aureus-associated septic shock. Int $\mathrm{J}$ Antimicrob Agents. 2013;41(3):255-60.

12. Rybak MJ, Vidaillac C, Sader HS, Rhomberg PR, Salimnia H, Briski LE, et al. Evaluation of vancomycin susceptibility testing for methicillin-resistant Staphylococcus aureus: comparison of Etest and three automated testing methods. J Clin Microbiol. 2013;51(7):2077-81. 
13. Diaz R, Afreixo V, Ramalheira E, Rodrigues C, Gato B. Evaluation of vancomycin MIC creep in methicillin-resistant Staphylococcus aureus infections-a systematic review and meta-analysis. Clin Microbiol Infect. 2018;24(2):97-104.

14. Adane ED, Herald M, Koura F. Pharmacokinetics of vancomycin in extremely obese patients with suspected or confirmed Staphylococcus aureus infections. Pharmacotherapy. 2015;35(2):127-39.

15. Ducharme MP, Slaughter RL, Edwards DJ. Vancomycin pharmacokinetics in a patient population: effect of age, gender, and body weight. Ther Drug Monit. 1994;16(5):513-8.

16. Bauer LA, Black DJ, Lill JS. Vancomycin dosing in morbidly obese patients. Eur J Clin Pharmacol. 1998;54(8):621-5.

17. Blouin RA, Bauer LA, Miller DD, Record KE, Griffen WO Jr. Vancomycin pharmacokinetics in normal and morbidly obese subjects. Antimicrob Agents Chemother. 1982;21(4):575-80.

18. Reynolds DC, Waite LH, Alexander DP, ReRyke CA. Performance of a vancomycin dosage regimen developed for obese patients. Am J Health Syst Pharm. 2012;69(11):944-50.

19. Leong JV, Boro MS, Winter M. Determining vancomycin clearance in an overweight and obese population. Am J Health Syst Pharm. 2011;68(7):599-603.

20. Moise PA, Culshaw DL, Wong-Beringer A, Bensman J, Lamp KC, Smith WJ, et al. Comparative effectiveness of vancomycin versus daptomycin for MRSA bacteremia with vancomycin MIC > $1 \mathrm{mg} / \mathrm{L}$ : a multicenter evaluation. Clin Ther. 2016;38(1):16-30.

21. Truong J, Veillette JJ, Forland SC. Outcomes of vancomycin plus a beta-lactam versus vancomycin only for treatment of methicillin-resistant Staphylococcus aureus bacteremia. Antimicrob Agents Chemother. 2018;62(2):e01445-17.

22. Claeys KC, Zasowski EJ, Casapao AM, Lagnf AM, Nagel JL, Nguyen CT, et al. Daptomycin improves outcomes regardless of vancomycin MIC in a propensity-matched analysis of methicillin-resistant Staphylococcus aureus bloodstream infections. Antimicrob Agents Chemother. 2016;60(10):5841-8.

23. Šíma M, Hartinger J, Cikánková $T$, Slanař O. Importance of vancomycin loading doses in intermittent infusion regimens. J Infect Chemother. 2018;24(4):247-50.

24. Álvarez O, Plaza-Plaza JC, Ramirez M, Peralta A, Amador CA, Amador R. Pharmacokinetic assessment of vancomycin loading dose in critically ill patients. Antimicrob Agents Chemother. 2017;61(8):e00280-17.

25. Truong J, Levkovich BJ, Padiglione AA. Simple approach to improving vancomycin dosing in intensive care: a standardised loading dose results in earlier therapeutic levels. Intern Med J. 2012;42(1):23-9.

26. Wesolek JL, McNorton K, Delgado G Jr, Giuliano CA. Effect of vancomycin initial dosing on time to systemic inflammatory response syndrome resolution in patients with methicillin-resistant Staphylococcus aureus bacteremia. J Chemother. 2018;30(2):101-6.

27. Rosini JM, Davis JJ, Muenzer J, Levine BJ, Papas MA, Comer D, et al. High single-dose vancomycin loading is not associated with increased nephrotoxicity in emergency department sepsis patients. Acad Emerg Med. 2016;23(6):744-6. 